\title{
Impact process of boulders on the surface of asteroid 25143 Itokawa- fragments from collisional disruption
}

\author{
A. M. Nakamura ${ }^{1}$, T. Michikami ${ }^{2}$, N. Hirata ${ }^{3}$, A. Fujiwara ${ }^{4}$, R. Nakamura ${ }^{5}$, M. Ishiguro ${ }^{6}$, H. Miyamoto ${ }^{7,8}$,

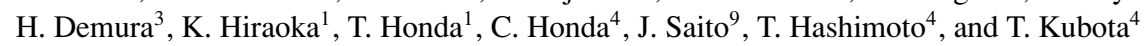 \\ ${ }^{1}$ Graduate School of Science, Kobe University, Kobe, 657-8501, Japan \\ ${ }^{2}$ Fukushima National College of Technology, Iwaki, Fukushima 970-8034, Japan \\ ${ }^{3}$ School of Computer Science and Engineering, University of Aizu, Aizuwakamatsu, Fukushima 965-8580, Japan \\ ${ }^{4}$ Institute of Space and Astronautical Sciences (ISAS), Japan Aerospace Exploration Agency (JAXA), \\ Sagamihara, Kanagawa 229-8510, Japan \\ ${ }^{5}$ National Institute of Advanced Industrial Science and Technology, Tsukuba 306-8568, Japan \\ ${ }^{6}$ Astronomy Department, Seoul National University, Seoul 151-747, Korea \\ ${ }^{7}$ The University Museum, University of Tokyo, Tokyo 113-0033, Japan \\ ${ }^{8}$ Planetary Science Institute, Tucson, AZ 85719, USA \\ ${ }^{9}$ School of Engineering, Tokai University, Hiratsuka, Kanagawa 259-1292, Japan
}

(Received November 3, 2006; Revised March 29, 2007; Accepted April 11, 2007; Online published February 12, 2008)

\begin{abstract}
The subkilometer-size asteroid 25143 Itokawa is considered to have a gravitationally bounded rubble-pile structure. Boulders appearing in high-resolution images retrieved by the Hayabusa mission revealed the genuine outcome of the collisional event involving the asteroid's parent body. Here we report that the boulders' shapes and structures are strikingly similar to laboratory rock impact fragments despite differences of orders of magnitude in scale and complexities of the physical processes. These similarities suggest the universal character of the process throughout the range of these scales, and the brittle and structurally continuous nature regarding the parent body of the boulders. The similarity was likely preserved because of relatively lesser comminuting processes acting on individual boulders; the close assemblages of similar appearing boulders (a boulder family) represent the impact destruction of boulders on the surface.
\end{abstract}

Key words: Asteroid, boulder, impact, fragmentation.

\section{Introduction}

The collisional growth and disruption of solid bodies take place over many magnitudes of scale, ranging from dust particles to planets. Small bodies in the solar system such as asteroids experience mutually direct and destructive collisions (Chapman and Davis, 1975). Asteroids are considered to have internal structures resulting from collisions that range from monolithic, fractured and shattered due to moderate impacts to gravitationally reagglomerated rubble piles following major disruption. The outcome of a collisional disruption depends largely on the mechanical property of the parent body (Holsapple et al., 2002; Richardson et al., 2002; Michel et al., 2003), that is, the transmission efficiency of the impact energy throughout the body (porosity) and the bonding strength between the constituent components of the body. However, only limited and indirect information about the mechanical property of asteroids has been available. Meteorites are the compositionally corresponding materials of asteroids, but our collection has probably suffered greatly from a selection effect against weaker materials due to dynamical pressure during atmospheric entry and transit. For example, analyses of the trajectory of a me-

Copyright (c) The Society of Geomagnetism and Earth, Planetary and Space Sciences (SGEPSS); The Seismological Society of Japan; The Volcanological Society of Japan; The Geodetic Society of Japan; The Japanese Society for Planetary Sciences; TERRAPUB teorite's fall have suggested that meter-class, stony, nearEarth asteroids (NEAs) have tensile strengths more than an order of magnitude lower than those measured for ordinary chondrites (Brown et al., 2004).

Laboratory studies on the impact process of asteroids have been undertaken for the past decades using light-gas gun and other facilities (Fujiwara et al., 1989; Holsapple et al., 2002). Although these experimental studies have provided quantitative data and insights into many aspects of centimeter-scale impact processes, difficulty in applying these findings to asteroids does exist due to the very limited scale of the experiments. The laboratory experiments have therefore been extended to the larger scales by numerical studies and scaling methods.

Asteroid 25143 Itokawa, about 500 meters long and $3.5 \times 10^{10} \mathrm{~kg}$ in mass, is considered to have a rubble-pile structure consisting of fragments from an earlier collisional disruption of a preexisting parent asteroid (Fujiwara et al., 2006). The size of the largest boulder (Yoshinodai) is onetenth of Itokawa itself and the number of boulders increases with decreasing size (Saito et al., 2006). Boulders larger than several meters in maximum width number in the hundreds. They cannot have been fully supplied by the craters on the current surface, but must have originated on the parent body or from the Itokawa-forming collisional event. Images of the boulders on Itokawa's surface with pixel resolu- 
tion of $\sim 6 \mathrm{~mm}$ to $\sim 70 \mathrm{~cm}$ taken by the Asteroid Multi-band Imaging CAmera (AMICA) provide us with the actual outcome of the collisional disruption event of the parent body of a subkilometer-size asteroid.

In Section 2, we report the morphological characteristics of the boulders on Itokawa and provide comparisons between the boulders and the laboratory impact fragments. In Section 3, we discuss the evidences for possible impact processes of boulders on the surface. Section 4 presents our conclusion. In this paper, we refer to apparently rootless rocks and features with distinctive positive relief larger than a few meters as "boulders" in accordance with a previous definition (Saito et al., 2006).

\section{Boulder Shape and Structure}

Boulders do not cover the surface of Itokawa uniformly. The boulder-dominated region is called "rough terrain", whereas the remainder is "smooth terrain" (Saito et al., 2006). Figure 1 shows the boulder-rich region (the rough terrain) on Itokawa's surface in contrast to the smooth terrain. While both angular and degraded boulders were observed on asteroid 433 Eros (Thomas et al., 2001), the boulders on Itokawa exhibit a wide spectrum of angularity and irregularity (Figs. 2(a)-(i)). Thin, flat-looking boulders were also seen, although they may be parts of bigger blocks that are mostly hidden beneath the surface. One of these with a spatula-like shape (Figs. 2(d), (e)) was observed in multiple images and its thickness-to-width ratio was determined to be $\sim 0.2$. A Similarly wide range of shapes is also seen in the fragments from laboratory impact disruptions. Figures 2(j)-(1) show fragments of centimeter-size collected after laboratory impacts. Basalt spheres of $6 \mathrm{~cm}$ in diameter and only $0.3 \mathrm{~kg}$ in mass were impacted by a 7 mm-diameter nylon projectile at $\sim 3.2 \mathrm{~km} / \mathrm{s}$ (Nakamura and Fujiwara, 1991; Nakamura, 1993; Nakamura et al., 2007). Fragments in Fig. 2(j) are angular and conical, which is characteristic of boulders in Fig. 2(b). The square fragments in Fig. 2(k) look similar to the boulders in Fig. 2(a). The irregularly shaped (wavy-shaped) fragment in Fig. 2(k) is similar to the one in Fig. 2(i).

The similarity in shape suggests a brittle and consolidated nature of the boulders' parent body, in which cracks could propagate. The thin shape is additional evidence suggestive of such a nature. Thin spall fragments are ejected at an impact event because of tensile fracture caused by a tensile wave reflected from a compressive wave at the free surface of the target. In other words, spallation occurs at the free surface when the compressive wave generated by an impact travels to the surface without being severely attenuated by pores and internal discontinuities in the target material along the way. The average ratio of the thickness to maximum length of the spall fragments from cratering experiments was 0.2 (Polanskey and Ahrens, 1990), in good agreement with the measured value of the spatula-like thin boulder, while the average ratio of the fragments from a disruption event is larger (0.45-0.5; Fujiwara et al., 1989; Giblin et al., 1998). The layered structure of the Yoshinodai boulder (Figs. 2(d), (e)) is also likely explained by spall failure similar to the core fragment shown in Fig. 2(1); however, another possible explanation may lie in the stratification of

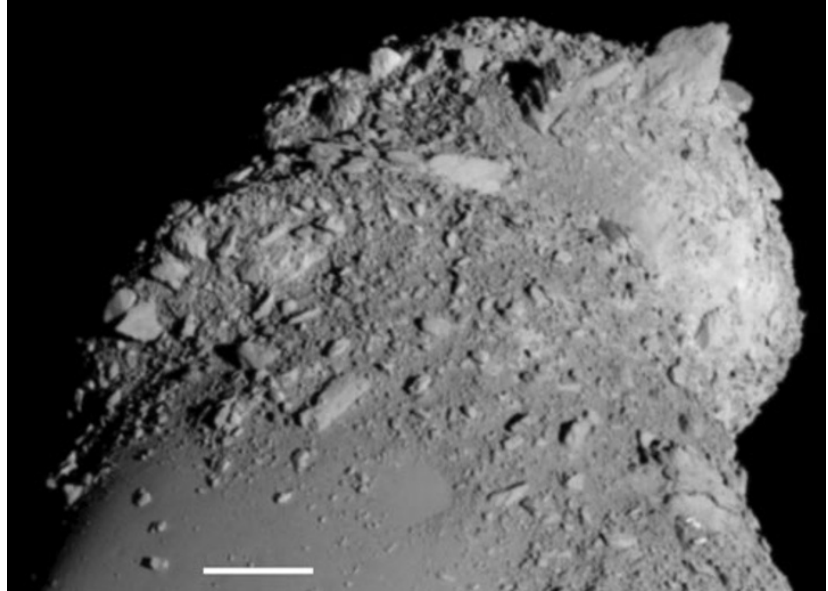

Fig. 1. View of a boulder-rich region on Itokawa (image no. ST_2481211873). At the upper left, the boulders are in a pile. Scale bar indicates $30 \mathrm{~m}$.

the parent body of the Yoshinodai boulder.

The most prominent features on the boulders are cracks and fractures (Figs. 2(b), (f)-(h)). A possible origin of the cracks and fractures is any process after the boulder was formed, for example, impact fracturing on Itokawa's surface (possibly the cracks on the boulder in Fig. 2(h), since small pieces near the boulder with a similar appearance could have been fragmented from the boulder). However, this is unlikely for most of the cracks of the boulders, since they are not linked with any crater-like depression expected for an impact site. Moreover, cracks are commonly observed in laboratory impact fragments (Fig. 2(k)) generated from originally homogeneous targets with no apparent surface cracks before impact disruption. These cracks also remained on fragments in numerical simulations of solid body disruption (Benz and Asphaug, 1994) and were caused by the growth of incipient microscopic flaws of the target body. Figure 3 illustrates the largest cracks reach a length up to about 0.8 times the maximum width of fragments, similar to the apparent cracks in the boulders on Itokawa. The fragments and boulders having cracks of lengths similar to their own sizes are in a state corresponding to "fully cracked" at that size scale (Housen and Holsapple, 1999). It is likely that the cracks on the boulder surfaces are mostly due to the impact event that created the boulders.

Although there is a difference of many orders of magnitude in the scale and complexity of the physical processes, these first-order similarities in shapes and structures of the boulders and the laboratory fragments bridge laboratory disruption of solid bodies (governed by the growth and coalescence of microscopic flaws) and the natural collisional disruption process. These similarities suggest a universal character of the process throughout these scales, and the brittle and structurally continuous nature of the parent body of the boulders (the parent body of boulders as well as the boulders themselves have considerable strength). The similarities encourage discussion on the impact process of the boulders based on our present understanding about the impact process of brittle materials. 

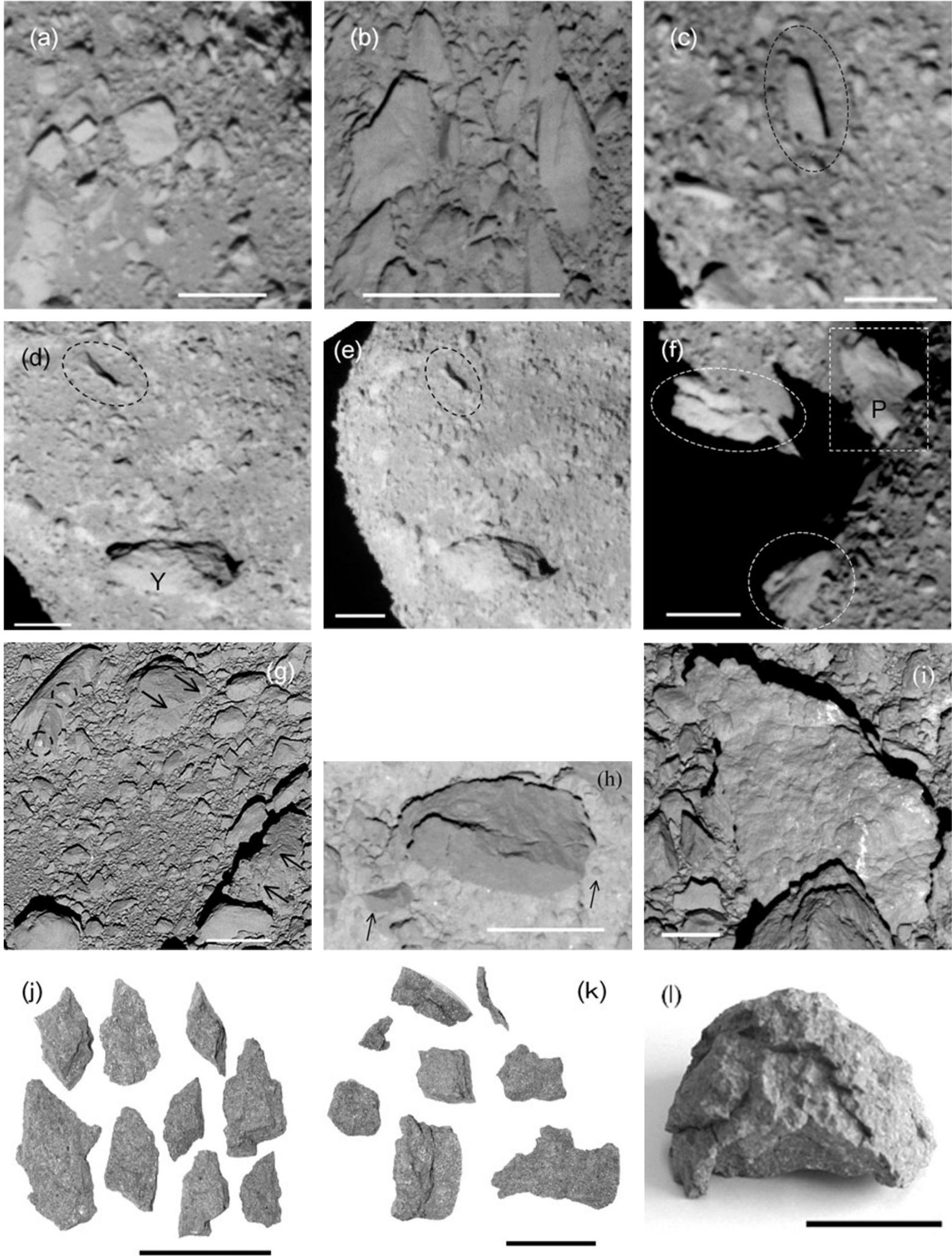

Fig. 2. Boulders on Itokawa's surface and fragments from laboratory impact disruption of 6-cm-diameter basalt spheres (Nakamura and Fujiwara, 1991; Nakamura, 1993). (a) Angular boulders at the neck region on the western side of the body (ST_2480981469). (b) Angular, conical boulders at the neck region on the eastern side of the body (ST_2530286817). (c) A flat-looking boulder at the head (ST_2490253205). (d) A very thin, spatula-shaped boulder (ST_2489696338). Y: Yoshinodai boulder (Demura et al., 2006). (e) The same boulder in a different image (ST_2481211873). (f) Boulders with cracks and fractures (ST_2482160259). P: Pencil boulder (Demura et al., 2006). (g) Boulders in a close-up image; meter-sized boulders with white spots (upper left) in dashed circles and linear and wavy cracks indicated by arrowheads (upper center and lower right, respectively) (ST_2544464441). Original lossless compressed image data were deconvolved by a point-spread function of the camera to restore the blur. (h) A boulder with a well developed crack. Small pieces at the arrowheads maybe fragmented from the boulder (ST_2539451609). (i) A highly irregularly shaped boulder (ST_2539437177). (j) Angular, conical fragments. (k) Fragments in various shapes. Some have well developed cracks on the surface. (l) A fragment from the central part of a spherical target. The surface was shaped by spallation. A fracture with a layered appearance developed. Scale bars (a-f) indicate $20 \mathrm{~m},(\mathrm{~g}) 2 \mathrm{~m}$, (h) and (l) $1 \mathrm{~m}$ based on the spacecraft altitude determined by LIDAR (Abe et al., 2006); (j-1), $2 \mathrm{~cm}$. 


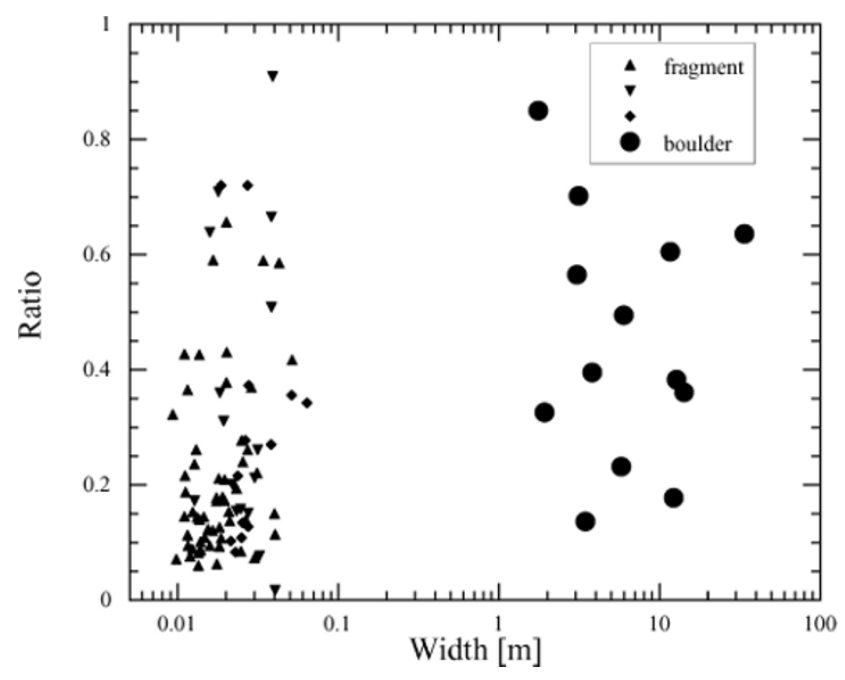

Fig. 3. Ratio of the maximum crack rectilinear length to the maximum width of boulders. The ordinate and abscissa axes show the ratio and the maximum width of boulders, respectively. Data were collected from images ST_2539423137, ST_2539429953, ST_2539437177, ST_2539444467, ST_2539451609, ST_2539467169, ST_2558581440, and ST_2559003068. Data from fragments in laboratory impact experiments (Nakamura and Fujiwara, 1991; Nakamura, 1993) are also shown for comparison.

\section{Boulder Impact Processes}

The impact process of the boulders on Itokawa's surface is mainly either high-velocity impacts of solid bodies or particles from interplanetary space into the boulders on the surface, or low-velocity impacts of the boulders onto Itokawa's surface, which is discussed in more detail below. The signature of the impact processes of the boulders other than cracks and fractures are white spots on the surface, probably due to interplanetary dust particle (IDP) bombardments (Figs. 2(g), 4(a)). A well developed fracture on the top surface of a boulder shown in Fig. 4(a), as well as the cracks on the boulder in Fig. 2(h), could also be due to IDP impacts. A pair of boulders (Fig. 4(b)) and a group of boulders or a boulder family (Fig. 4(c)) indicate a more advanced state of impact destruction, although a possibility exists that they are merely tips of large buried boulders. As described previously, since both the spatula-shaped thin boulder and the Yoshinodai boulder could be the product of spallation, the spatula-shaped boulder may be fragmented from the surface of Yoshinodai. The fact that the spatula-shaped boulder is lying beside the major defect of the Yoshinodai boulder supports this conjecture (Figs. 2(d), (e)). The boulders in Fig. 4(c) are examples of higher degree of destruction; two groups of three pieces of boulders with apparently similar albedo occur closely together, and may have originated from one or two boulder (s) of at least $20 \mathrm{~m}$ in diameter.

\subsection{High-velocity impact into the boulders}

If the boulder pair and family are remnants of the disruption of larger boulders by impactors from interplanetary space, an impactor diameter of $\sim 0.4 \mathrm{~m}$ is required to break the $20 \mathrm{~m}$ boulder into pieces with the largest remnant mass being half of the original; this assumption is based on adopting an intermediate value for the specific energy density needed for such disruption from those derived in previous studies $\left(Q^{*}=10^{2} \mathrm{~J} / \mathrm{kg}\right.$, Holsapple et al., 2002) and the mean collision velocity in the main asteroid belt $(\sim 5 \mathrm{~km} / \mathrm{s}$; Bottke et al., 1994). A $\sim 0.4$ m-diameter impactor would excavate a crater $\sim 12 \mathrm{~m}$ in diameter if the impactor hits a homogeneous, semi-infinite surface of typical S-type asteroids (Richardson et al., 2005). The parent boulders of the boulder families shielded the Itokawa surface from cratering excavation due to such impactors by consuming their energy and momentum in fragmentation and fragment dispersion, that is, by armoring the surface from cratering with boulders if that did occur (Chapman et al., 2002).

Based upon the hypothesis of the boulder pairs and families are remnants of the armoring process, it is inferred that some fraction of the fragments was retained or reaccumulated on the surface despite Itokawa's very low gravitational field. The fraction is known to be highly dependent upon the strength of the surface in the case of cratering events (Housen, 1992; Michikami et al., 2007). The boulder material on Itokawa's surface seems to have much greater strength than unconsolidated material, based on the shape and structure of the boulders as discussed above. The fraction of the retained and reaccumulated mass is accordingly expected to be very small ( $\sim 1 \%$ or less), and such an impact process on Itokawa's surface is in principle erosive. However, this fraction might be larger in the case of impacts into boulders. Boulders deeply buried in piles of other boulders (e.g., Figs. 4(a) and possibly 4(b)) could be relatively easily retained on the surface against impact fracturing because part of the impact energy would be transferred to the surrounding boulders. Meanwhile, an impact into a boulder on Itokawa's surface would produce ejecta moving downward in the direction of Itokawa's interior, and such ejecta may have a greater chance of being retained on the surface. 3.2 Low-velocity impact of the boulders onto Itokawa's surface

If any boulders large enough to be the parent of the boulder pairs or families were to hit Itokawa's surface with a velocity sufficiently low for at least some portions to remain on the surface, this would constitute an alternative process for the origin of the pairs and families. Assuming the same $Q^{*}\left(=10^{2} \mathrm{~J} / \mathrm{kg}\right)$ as above, the impact velocity for the survival of the nonnegligible part of the parent boulder would be less than a few tens of $\mathrm{m} / \mathrm{s}$. Boulders associated with circular depressions in the Muses Sea may be remnants of such low-velocity impact events (Saito et al., 2006), which would have occurred after the development of the current Muses Sea.

Two types of low-velocity impact can occur: the secondary impact of ejecta blocks from a primary impact on the surface, and the impact of remnant small ejecta from the Itokawa-forming event. The former process has two difficulties regarding the origin of the boulder families, especially the large ones shown in Fig. 4(c). First, boulders tens of meter in diameter could hardly have been ejected from any of the craters now on the surface of Itokawa based on the empirical relationship between the largest boulder and the source crater diameter (Thomas et al., 2001; Fujiwara et al., 2006; Saito et al., 2006). Second, the maximum possible velocity onto the surface, which is constrained by the escape velocity of Itokawa, could hardly break the boulders. Given a velocity of $0.1 \mathrm{~m} / \mathrm{s}$, the energy density $(Q)$ for the 

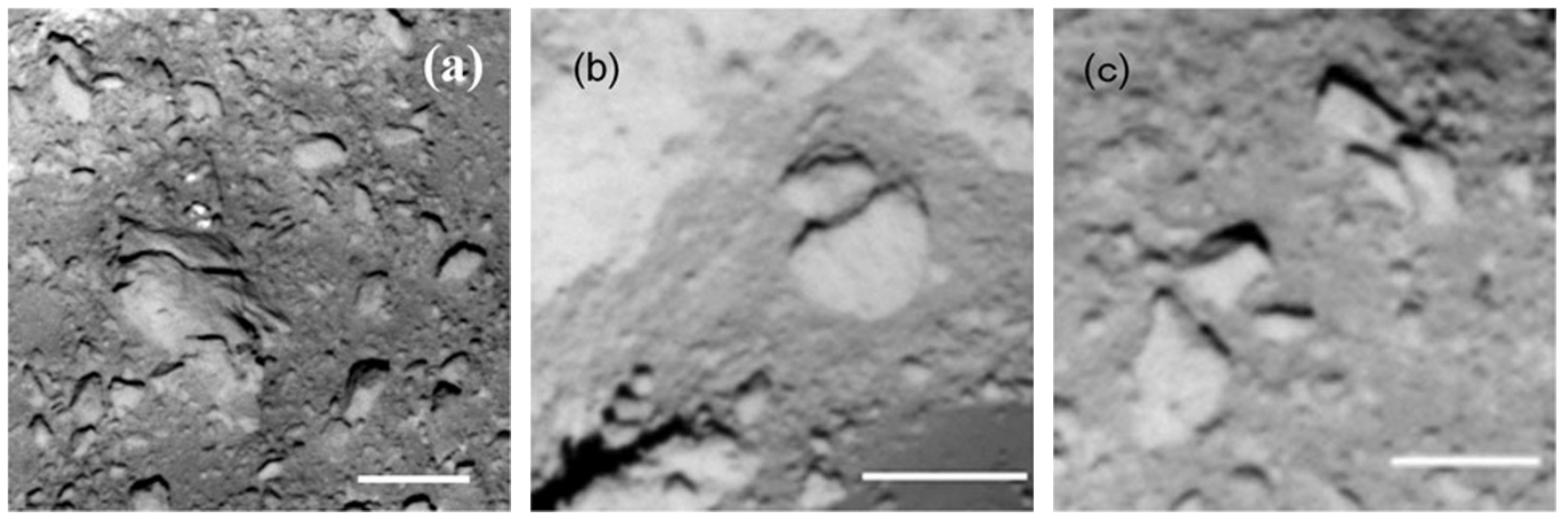

Fig. 4. Evidence of possible impact processes on Itokawa's surface. (a) Fractured boulder in Tsukuba Region*1 (Demura et al., 2006) (ST_2494934387). White spots are also shown. (b) Pair of boulders named Mountainview boulders (Demura et al., 2006) (ST_2506656195). (c) Two groups of boulders (ST_2471012186). Scale bars (a-c) indicate $20 \mathrm{~m}$, based on the spacecraft's altitude determined by LIDAR (Abe et al., 2006).

breakage of the parent boulder becomes $5 \times 10^{-3} \mathrm{~J} / \mathrm{kg}$, a value far less than the $Q^{*}$ found for centimeter-scale ice $(41 \mathrm{~J} / \mathrm{kg})$, rock $\left(6.8 \times 10^{2} \mathrm{~J} / \mathrm{kg}\right)$ (Hartmann, 1978) and weakly sintered porous glass beads (a few $\mathrm{J} / \mathrm{kg}$ for those having a compressive strength of $\sim 1 \mathrm{MPa}$ ) (Setoh et al., 2007). Due to the size dependence of both static and impact strength, $Y \propto L^{-3 / m}$, where $Y, L$, and $m$ denote strength, boulder size, and flaw-size distribution exponent or Weibull modulus, respectively (Benz and Asphaug, 1994; Housen and Holsapple, 1999; Asphaug et al., 2002; Nakamura et al., 2007), and the $Q^{*}$ needed for breakup decreases with increasing size of the parent body of the boulders. Nevertheless, the value of $5 \times 10^{-3} \mathrm{~J} / \mathrm{kg}$ is still too small for a continuous brittle material, if a typical value of $m$ (down to $\sim 6$ ) for rocks in the literature is taken into account. Detailed evaluation of the latter process will require intensive study (Davis et al., 1996; Michel et al., 2003) of time evolution of collisional frequency and impact velocity of the remnant ejecta. Only limited impact velocity could explain the facts that the members of the boulder families could have had only very small residual velocity in order to remain on the surface in close proximity to each other, and the observation that no apparent craters are associated with the large boulder families in Fig. 4(c).

\subsection{Impact history of the boulders}

A fundamental question is to what extent the boulders presently on Itokawa's surface differ from what they were at Itokawa's origin, when the parent body was disrupted and the major reagglomeration was completed or the head-body system of Itokawa was formed (Fujiwara et al., 2006). At the beginning, the rough and smooth terrains were hardly separated; boulders probably covered the whole surface like the boulder-rich region in Fig. 1. A typical collisional survival lifetime of a meter-sized isolated body in the asteroid mainbelt is estimated to be $7 \mathrm{Myr}$ (O'Brien et al., 2005) or 14 Myr (Bottke et al., 2005) by current models of collisional evolution of asteroids. This is shorter than the collisional lifetime estimate for an Itokawa-sized body (30-100 Myr), implying the possibility of one or more cycles of surface

\footnotetext{
${ }^{* 1}$ This is a temporal designation before the region is named by the International Astronomical Union (IAU).
}

boulders disruption. Consequently, some of the boulders now exposed on Itokawa's surface may have been shielded by the other boulders at Itokawa's first reagglomeration and exposed some time thereafter to be roasted by space weathering (Ishiguro et al., 2007).

\section{Summary}

Asteroid 25143 Itokawa is the first body explored by a spacecraft that appears to have a rubble-pile structure, in which boulders of various sizes constitute a gravitationally bound body. The high-resolution images of boulders on the Itokawa show striking similarities between the constituent meter- and decameter-sized boulders of this intriguing asteroid and the small-scale fragments from laboratory experiments. This thus appears to constitute a bridge between the laboratory destruction of solid bodies (governed by the growth and coalescence of inherent microscopic flaws) and natural collisional destruction processes, although a difference of many orders of magnitude exists in the scale and complexity of the physical processes. The similarity also suggests that the brittle and structurally continuous nature of the parent body of the boulders as well as the boulders themselves have considerable strength.

White spots on the boulders, apparent fractures, and groups of boulders or boulder families show different degree of the impact process occurring with the boulders. These are probably due to high-velocity impacts of solid bodies or particles from interplanetary space. In this regard, some of the boulders presently on the surface were shielded by other boulders and subsequently exposed to interplanetary space.

Acknowledgments. We thank the continuous efforts and support of the mission operation and spacecraft team of the Hayabusa project at ISAS/JAXA. The technical staff at the NEC Toshiba Space Systems (NTSpace) is also greatly acknowledged for their dedicated work during the manufacturing and the ground tests of the AMICA instrument. We also express our thanks to all the members of the AMICA team and Hayabusa Science Team for their cooperation and encouragement of the AMICA mission. This research was supported by Kobe University through "The 21st Century COE Centers of Excellence Program of the Origin and Evolution of Planetary Systems". 


\section{References}

Abe, S. et al., Mass and local topography measurements of Itokawa by Hayabusa, Science, 312, 1344-1346, 2006.

Aspahug, E., E. V. Ryan, and M. T. Zuber, Asteroid interiors, in Asteroids III, edited by W. F. Bottke Jr, A. Cellino, P. Paolicchi, and R. P. Binzel, 463 pp., Univ. Arizona Press, Tucson, 2002.

Benz, W. and E. Asphaug, Impact simulations with fracture, I-Method and tests, Icarus, 107, 98-116, 1994.

Bottke Jr., W. F., M. C. Nolan, R. Greenberg, and R. A. Kolvoord, Velocity distributions among colliding asteroids, Icarus, 107, 255-268, 1994.

Bottke Jr., W. F. et al., Linking the collisional history of the main asteroid belt to its dynamical excitation and depletion, Icarus, 179, 63-94, 2005.

Brown, P. et al., The orbit, atmospheric dynamics, and initial mass of the Park Forest meteorite, Meteorit. Planet. Sci., 39, 1781-1796, 2004.

Chapman, C. R. and D. R. Davis, Asteroid collisional evolution-Evidence for a much larger early population, Science, 190, 553-556, 1975.

Chapman, C. R., W. J. Merline, P. C. Thomas, and J. Joseph, Impact history of Eros: Craters and boulders, Icarus, 155, 104-118, 2002.

Davis, D. R. et al., The formation and collisional/dynamical evolution of the Ida/Dactyl system as part of the Koronis family, Icarus, 120, 220 230, 1996.

Demura, H. et al., Pole and global shape of 25413 Itokawa, Science, 312, 1347-1349, 2006.

Fujiwara, A. et al., The rubble-pile asteroid Itokawa as observed by Hayabusa, Science, 312, 1330-1334, 2006.

Giblin, I. et al., The properties of fragments from catastrophic disruption events, Icarus, 134, 77-122, 1998.

Hartmann, W. K., Planet formation: Mechanism of early growth, Icarus, 33, 50-61, 1978 .

Holsapple, K., I. Giblin, K. Housen, A. Nakamura, and E. Ryan, Asteroid Impacts: Laboratory Experiments and Scaling Laws, in Asteroids III, edited by W. F. Bottke Jr, A. Cellino, P. Paolicchi, and R. P. Binzel, 443 pp., Univ. Arizona Press, Tucson, 2002.

Housen, K. R., Crater ejecta velocities for impacts on rocky bodies, LPSC XXIII abst., 555-556, 1992.

Housen, K. R. and K. A. Holsapple, Scale effects in strength-dominated collisions of rocky asteroids, Icarus, 142, 21-33, 1999.

Ishiguro, M. et al., Global mapping of the degree of space weathering on asteroid 25143 Itokawa by Hayabusa/AMICA observations, Meteoritics
\& Planet. Sci., 2007 (in press).

Michel, P., W. Benz, and D. C. Richardson, Disruption of fragmented parent bodies as the origin of asteroid families, Nature, 421, 608-611, 2003.

Michikami, T., K. Moriguchi, S. Hasegawa, and A. Fujiwara, Ejecta velocity distribution for impact cratering experiments on porous and low strength targets, Planet. Space Sci., 55, 70-88, 2007.

Nakamura, A. and A. Fujiwara, Velocity distribution of fragments formed in a simulated collisional disruption, Icarus, 92, 132-146, 1991.

Nakamura, A. M., Laboratory studies on the velocity of fragments from impact disruptions, ISAS Report, 651, 1993.

Nakamura, A. M., P. Michel, and M. Setoh, Weibull Parameters of Yakuno Basalt Targets Used in Documented High-Velocity Impact Experiments, J. Geophys. Res. (Planets), 112, 10.1029/2006JE002757, 2007.

O'Brien, D. P. and R. Greenberg, The collisional and dynamical evolution of the main-belt and NEA size distributions, Icarus, 178, 179-212, 2005.

Polanskey, C. A. and T. J. Ahrens, Impact spallation experiments: Fracture patterns and spall velocities, Icarus, 87, 140-155, 1990.

Richardson, D. C., Z. M. Leinhardt, H. J. Melosh, W. F. Bottke Jr., and E. Asphaug, Gravitational Aggregates: Evidence and Evolution, in Asteroids III, edited by W. F. Bottke Jr, A. Cellino, P. Paolicchi, and R. P. Binzel, 501 pp., Univ. Arizona Press, Tucson, 2002.

Richardson Jr., J. E., H. J. Melosh, R. J. Greenberg, and D. P. O'Brien, The global effects of impact-induced seismic activity on fractured asteroid surface morphology, Icarus, 179, 325-349, 2005.

Saito, J. et al., Detailed images of asteroid 25143 Itokawa from Hayabusa, Science, 312, 1341-1344, 2006.

Setoh, M., A. M. Nakamura, N. Hirata, K. Hiraoka, and M. Arakawa, Collisional disruption of weakly sintered porous targets at low-impact velocities, Earth Planets Space, 59, 319-324, 2007.

Thomas, P. C., J. Veverka, M. S. Robinson, and S. Murchie, Shoemaker crater as the source of most ejecta boulders on the asteroid 433 Eros, Nature, 413, 394-396, 2001.

A. M. Nakamura (e-mail: amnakamu@kobe-u.ac.jp), T. Michikami, N Hirata, A. Fujiwara, R. Nakamura, M. Ishiguro, H. Miyamoto, H. Demura, K. Hiraoka, T. Honda, C. Honda, J. Saito, T. Hashimoto, and T. Kubota 